\title{
BIOAEROSOL PROPERTY AND VIABILITY AFFECTED BY VARIOUS ENVIRONMENTAL FACTORS
}

\author{
YONG-LE PAN ${ }^{1}$, AIMABLE KALUME $^{1}$, SEAN KINAHAN $^{2}$, MATTHEW TEZAK $^{2} \&$ JOSHUA SANTARPIA $^{2,3}$ \\ ${ }^{1}$ US Army Research Laboratory, USA \\ ${ }^{2}$ Sandia National Laboratories, USA \\ ${ }^{3}$ National Strategic Research Institute and University of Nebraska Medical Center, USA
}

\begin{abstract}
The monitoring of air pollution, especially the detection and characterization of biological aerosols (bioaerosols) in the atmosphere continues to be a challenging task. Most biosensors rely on the presence of specific molecules, such as antigens on the surface, DNA sequences, or the common fluorescents tryptophan, flavins, or reduced form of nicotinamide adenine dinucleotide (NADH). However, the detection signatures from either of these technologies can change significantly when the bioaerosol is released into the atmosphere, and the observed changes are strongly dependent upon the environmental conditions. In developing bioaerosol detection and characterization methods, researchers must account for the potential changes in their physical, chemical, and biological properties caused by various atmospheric conditions. The experimental results presented here show how the fluorescence spectral profile and intensity, the viability, and the PCR signature of bioaerosols, in particular for the vegetative bacteria Escherichia coli, change with time in the presence of one, or combinations of two, three, or four of the following variables: relative humidity $<30 \%$ or $\sim 75 \%$, ozone $\sim 100 \mathrm{ppb}, \boldsymbol{\alpha}$-pinene $\sim 5 \mathrm{ppb}$, toluene $\sim 45 \mathrm{ppb}$, and simulated solar ultra-violet light illumination with the typical levels in common atmospheric constituents and meteorological conditions. Large changes have been observed, e.g. UV fluorescence intensity dropped to be less than $1 / 10$ of its initial value and the ratio of UV/visible fluorescence intensity flipped from 2 to $1 / 2$ within $3 \mathrm{~h}$. These changes could happen on a typical day in any city or suburban area. Recording data of the ageing processes measured here should be very useful in developing biosensors and monitoring air pollution.
\end{abstract}

Keywords: Age process, Bioaerosol, Environmental factors, Fluorescence spectra, PCR signature, Viability.

\section{INTRODUCTION}

Airborne aerosol particles, including bioaerosol particles, play a very important role in the atmosphere, where their physical, chemical, and biological properties, as well as their interactions with the environment have significant impacts on the climate, environment, and human health [1]-[3]. 'Primary biological aerosols (PBA), in short bioaerosols, are a subset of atmospheric particles, which are directly released from the biosphere into the atmosphere. They comprise living and dead organisms (e.g. algae, archaea, bacteria), dispersal units (e.g. fungal spores and plant pollen), and various fragments or excretions (e.g. plant debris and brochosomes. PBA particle diameters range from nanometres up to about a tenth of a millimetre' [4]. The properties and viability of bioaerosols in the atmosphere have shown significant changes as they interact with the local atmospheric environment. These changes of bioaerosol particles in the atmosphere could be resulting from oxidization by ozone or $\mathrm{OH}$ radicals, hydration or desiccation due to fluctuations in relative humidity $(\mathrm{RH})$, degradation by solar irradiation or light-induced photochemical reactions, agglomeration with other aerosols and interactions with secondary organic aerosols (SOA), free radicals, volatile organic compound (VOC), semi-VOC, or inorganic gas-phase compounds (e.g. NOx and SOx) [5] [15]. There are some studies about the total fluorescence changes for fungal spores as they exposed to flowing air in cultures of different ages [10]; about the changes of viability and 
inactivation, absorption and fluorescence for bacteria and aromatic amino acids in bulk samples after exposure to ozone [11]-[12]; and about the change of fluorescence and viability for bioaerosols suspended in air while they interact with ozone in different relative humidities [13]-[15]. However, there is limited study about the effects of these 'open air factors (OAF)' to bioaerosols suspended in real atmosphere. In the troposphere, particularly in areas impacted by anthropogenic emissions, the trace chemical composition can be quite complex and many other compounds, in addition to ozone and relative humidity, may affect the properties of bioaerosols. The changes of bioaerosols exposed to more complex mixtures of trace gasses (e.g. $\mathrm{O}_{3}$, radical species and VOCs) under different meteorological conditions (e.g. solar irradiation, RH and temperature) requires further study. In order to improve the ability for monitoring air pollution, detecting and characterizing bioaerosols, taking the advantage of using existing instruments, and better understanding the atmosphere, we explored further for the changes of fluorescence spectra, viability and the sensitivity of polymerase chain reaction (PCR) detection of bioaerosols under laboratory-generated complex chemical ageing processes in an improved rotating drum in this paper.

\section{MATERIAL AND METHODS}

A general schematic of the experimental setup is displayed in Fig. 1, the whole apparatus is composed of a rotating reaction drum chamber, an aerosol generator, an UV aerodynamic particle sizer (UV-APS), a single particle fluorescence spectrometer (SPFS), an all-glass impinger (AGI) for sample collection, and equipment to generate, monitor and control the ozone, RH, UV light simulated solar irradiation and two model atmospheric VOCs ( $\alpha$-pinene or toluene). The VOC compounds were chosen to be representative of both biogenic ( $\alpha$-pinene) and anthropogenic (toluene) compounds that are commonly attributed to the formation of secondary organic aerosol in the atmosphere. Concentration/intensity of ozone, water vapour, UV light irradiation, and the two VOCs can be controlled individually, so they can be introduced alone or together to examine their effects individually, or in combination. The chamber has a $400 \mathrm{~L}$ volume, corresponding to a radius of 15 inches and a length of 36 inches; it was used to ensure that there is sufficient volume for intermittent sampling throughout the ageing period of up to several hours. Dry laboratory compressed air, after removing residual volatile compounds and particulates by a carbon and high-efficiency particulate arresting (HEPA) filter, was used to supply clean dry air, humid air (through a deionized (DI) water bubbler), ozone-laden air, VOC-evaporation laden air, and aerosol generation air. Each of these air flows was metred at a target flow rate via mass flow controllers (MFC, Alicat Scientific) and controlled through a LabVIEW interface. Teflon-coated stainless-steel was used as the inert material of the entire drum to avoid the inadvertent generation of static charge during the rotation of the drum, other drum materials that are involved in rotation were selected to ensure that no plastic on plastic contact is made during rotation. The Teflon coating also limits the reactivity of the chamber walls to improve observations inside the drum.

Chemically conditioned air (ozone, RH, VOCs) can be introduced into the chamber by two methods. First, by introducing through the ports labelled inlets (Fig. 1), which allows better mixing and directly replaces air in the drum with conditioned air. This method is used to precondition the drum to the target chemical concentration and humidity quickly. Second, conditioned air can travel along a 2-inch diameter perforated tube, lined with a PTFE-membrane, which acts as a gas permeable conditioning system. This approach is utilized during testing, to allow mixing of gaseous species through diffusion, while minimizing impact to the aerosol concentration either by dilution or disruption to the rotational flow. Direct injection and sampling lines both have end conical reducers to limit the disrupting injecting or sampling air velocity by increasing the cross-sectional area. 


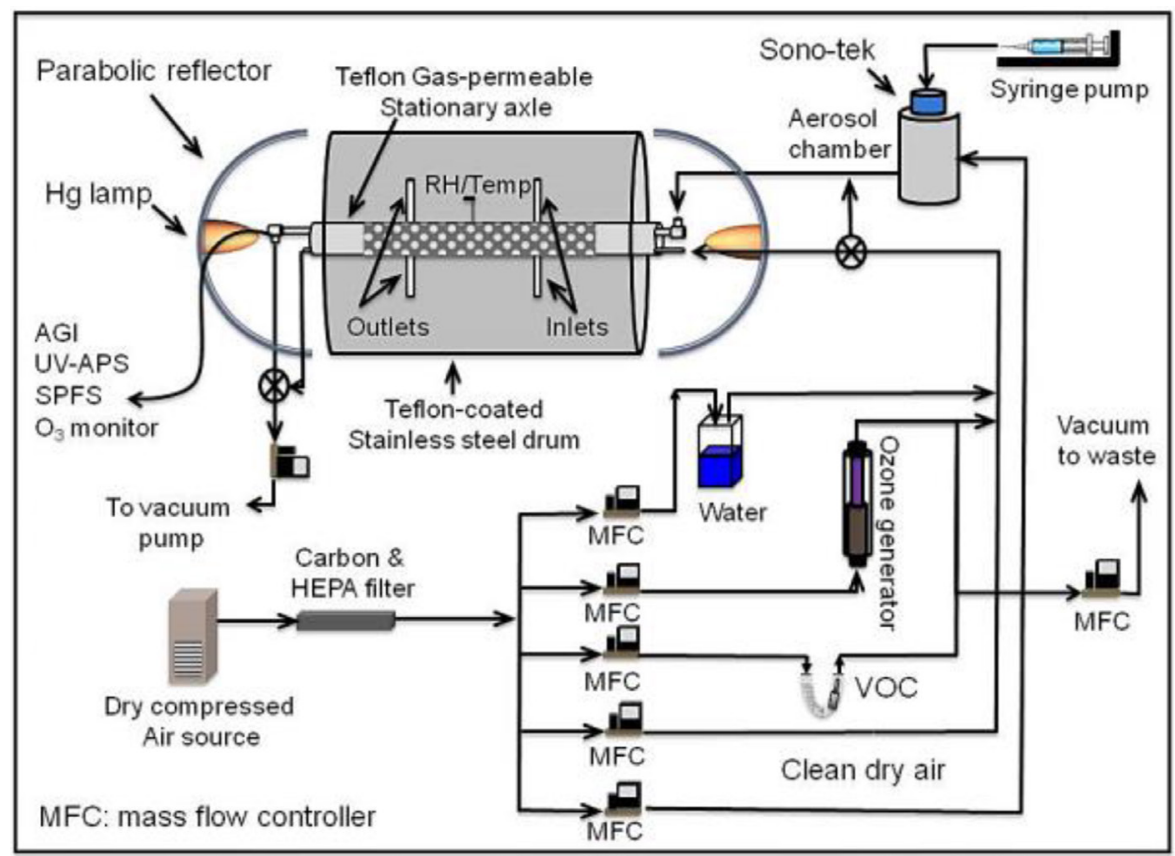

Figure 1: The schematic of the experimental setup. The scale of the axel relative parts of the rotating drum is exaggerated to show the details of the air and aerosol flow paths and different monitors.

During the test process, the drum was rotated at $1 \mathrm{rpm}$ to extend the settling time of the particles in the chamber. Particle retention in the drum was measured to be as good as $20 \%$ of the injected aerosols below $2 \mu \mathrm{m}$ for up to $5 \mathrm{~h}$. Figure 2 shows a typical concentration distributions of bioaerosol Bacillus thuringiensis Al Hakam (B.t.) vary with aerodynamic particle size at different time lapses, which were recorded at a half hour intervals, after released
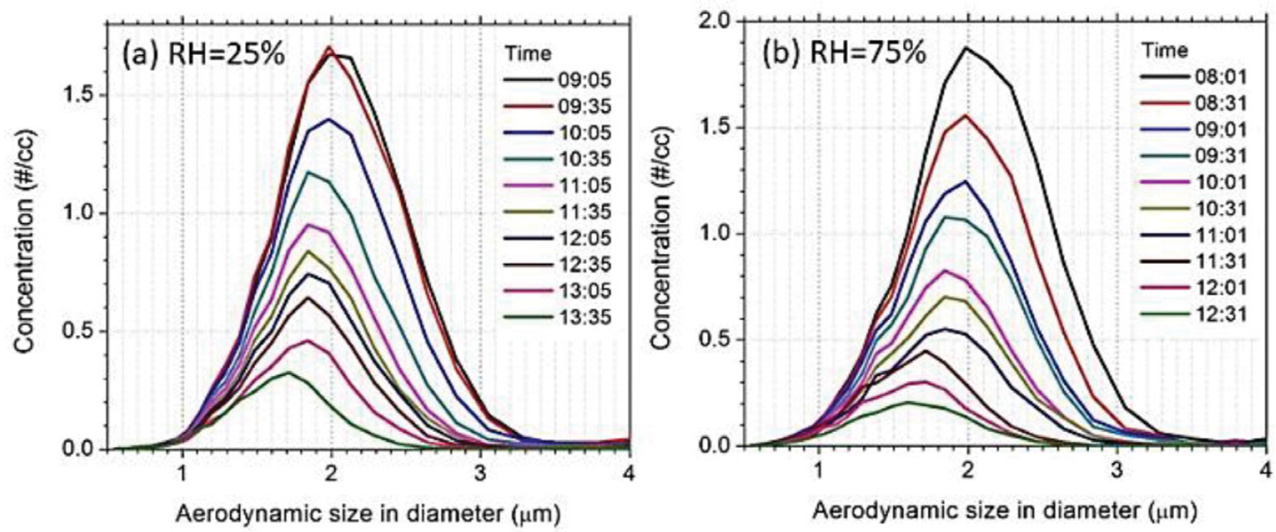

Figure 2: Concentration distributions of bioaerosol B.t. Al Hakam vary with aerodynamic particle size at different time lapses after released into the rotating drum under (a) low relative humidity $(20 \%)$ and (b) high relative humidity $(\sim 75 \%)$. 
into the rotating drum. Figures $2 \mathrm{a}$ and $2 \mathrm{~b}$ were injected into the chamber with a conditional chamber RH at $\sim 20 \%$ and $\sim 75 \%$, respectively. In both low and high RH conditions, particle concentration got lower and lower mostly due to the physical loss, the mode concentration kept shifting to a smaller size from $2 \mu \mathrm{m}$ to about $1.5 \mu \mathrm{m}$ within $4.5 \mathrm{~h}$, while the particles have a bigger lose and mode size shift in high RH than in low RH.

- Escherichia coli (E. coli) K-12 strain, Bacillus thuringiensis (B.t.) Al Hakam, Yersinia rohdei and MS2 bacteriophage were chosen to be the testing materials, particularly the experimental results from $E$. coli are presented in this article.

- The ageing process of the resting bioaerosol particles in the reaction chamber was characterizing by the ultra-violet (UV) laser-induced fluorescence spectra measured every half hour, also bacterial viability and PCR from the beginning and the end collected by the AGI sampler. The fluorescence spectra from single individual bioaerosol particles from the rotating drum at each measuring time points throughout each experiment were measured by the single particle fluorescence spectrometer (SPFS) [13]-[15]. It provided dispersed fluorescence spectra covered $280-560 \mathrm{~nm}$ excited by a 263-nm laser pulse and 380-650 nm excited by a 351-nm laser pulse associated with an elastic scattering particle size. Meanwhile an ultraviolet aerodynamic particle sizer (UV-APS, Model \#3312, TSI Inc.) measures the total fluorescence $(430-580 \mathrm{~nm})$ of aerosols excited by a $355 \mathrm{~nm}$ laser and reports the aerodynamic size of the aerosol between 0.523 and $20 \mu \mathrm{m}$, it supplies the aerosol concentration and size distribution during each test to normalize biological losses for physical losses (described below). Both the SPFS and UV-APS sampled from the chamber at $1 \mathrm{Lpm}$ during the measurements. For the aerosol samples, which were collected at the beginning and end of each test by an AGI-30s with $20 \mathrm{~mL}$ of phosphatebuffered saline, were analysed for bacterial viability and further analysed by PCR. To determine viability, the AGI-30 samples were serially diluted in PBS and plated in triplicate on 3M Petrifilm Aerobic Count Plates and LB agar to determine the equivalent spore/cell concentration. The Primers and probes for PCR signal are determined based on an existing published K-12 specific assay [16].

Finally, quantification by viable counts and PCR were normalized to particle concentrations in the drum, to compensate for the varying levels of particle loss depending on the test conditions, and the sample time. Meanwhile, the mass concentration of aerosols was used instead of number of concentration for higher reliable viability calculation. Therefore, the log decay rates normalized to aerosol concentration was calculated as,

$$
\left[\log _{10}\left(\frac{C_{0 A G I}}{n_{0}}\right)-\log _{10}\left(\frac{C_{1 A G I}}{n_{1}}\right)\right] / t_{1}
$$

where aerosol concentration $C$ is determined by PCR or plating, total aerosol concentration $n$ is determined by UV-APS; $t$ is the time point for second AGI sampling, which is equal to the ageing time of aerosol within the chamber; and the notation 0 and 1 indicates the initial and final aerosol sampling time point, respectively.

In the experiment, the variables were applied to the chamber either one by one individually, or in the combination of two, three, or four of them in observing the ageing process, both a zero and a controlled concentration of each variables were chosen to perform the experiment. In total 24 possible test conditions we used. Each of these conditions was repeated three to 
four times. Each test lasted at least 3-4.5 h, not including the time required for conditioning and purging the chamber. For the tests that had the contribution from VOC, only one of the two VOCs (either toluene or $\alpha$-pinene) was applied. During each test, bioaerosol were generated for approximately $10 \mathrm{~min}$ to reach a particle concentration of 300 particles $/ \mathrm{cm}^{3}$ in the chamber. Immediately following chemical conditioning and aerosol generation, initial measurements were made with the UV-APS, SPFS, and an 5 min. initial sample was collected with the AGI-30 at $12.5 \mathrm{Lpm}$. Throughout each test, UV-APS and SPFS took measurements every 30 min. UV-APS samples were collected for 3 min using a 30-s sampling cycle. The SPFS recorded 200 fluorescence spectra from individual particles for both $263-\mathrm{nm}$ and 351-nm excitations associated with its size in each measurement. Once aerosol was drawn from the chamber, its population was effectively diluted due to the influx of the clean particle-free air to maintain pressure.

\section{RESULTS AND DISCUSSIONS}

\subsection{Biological Signature Ageing}

Figures 3 and 4 summarize the biological ageing results of these experiments in terms of the corresponding log change rate (defined in eqn (1)) of viability and PCR sensitivity for aerosolized $E$. coli cells under the different experimental conditions. Changes in the viability of collected aerosol particles and changes in the PCR sensitivity were examined and normalized against physical losses in the chamber, as measured by the UV-APS.

Figures 3 and 4 show that $E$. coli exhibits significant change differences between ageing conditions. Mostly the changes in PCR detection for E. coli were not in good correlation with the changes in viability except in a few cases. For instance, high humidity demonstrated no significant ageing, alone or in combination with VOCs. However, UV light, high RH with

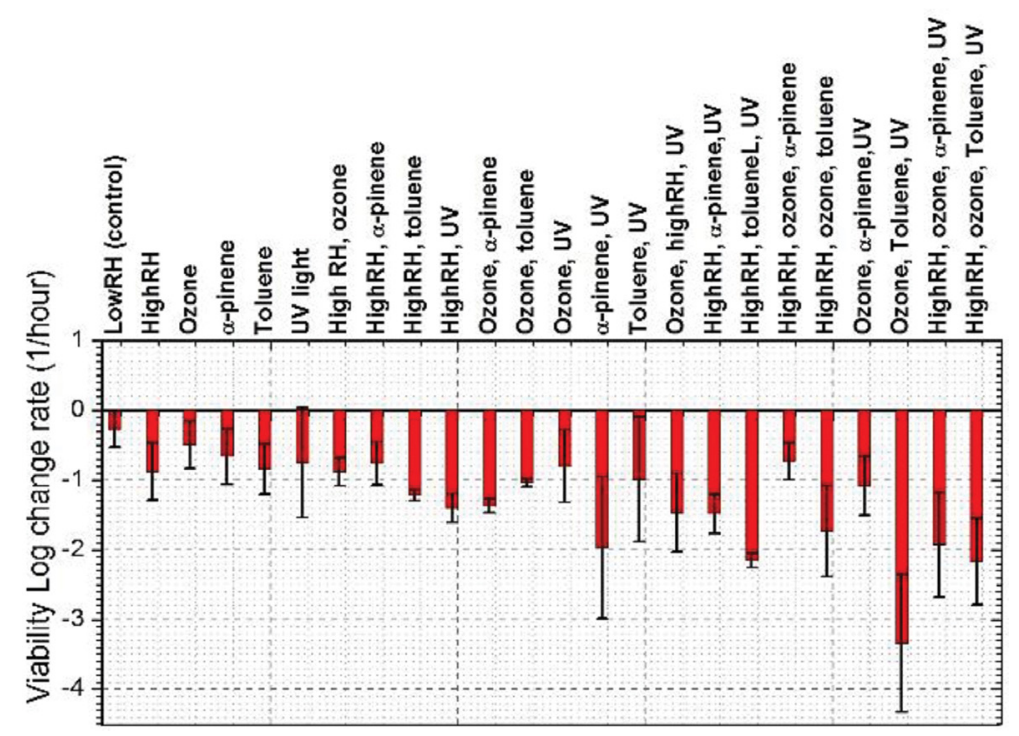

Figure 3: Log change rate (defined in eqn (1)) of viability for aerosolized $E$. coli cells under the different experimental conditions within the reaction chamber. 


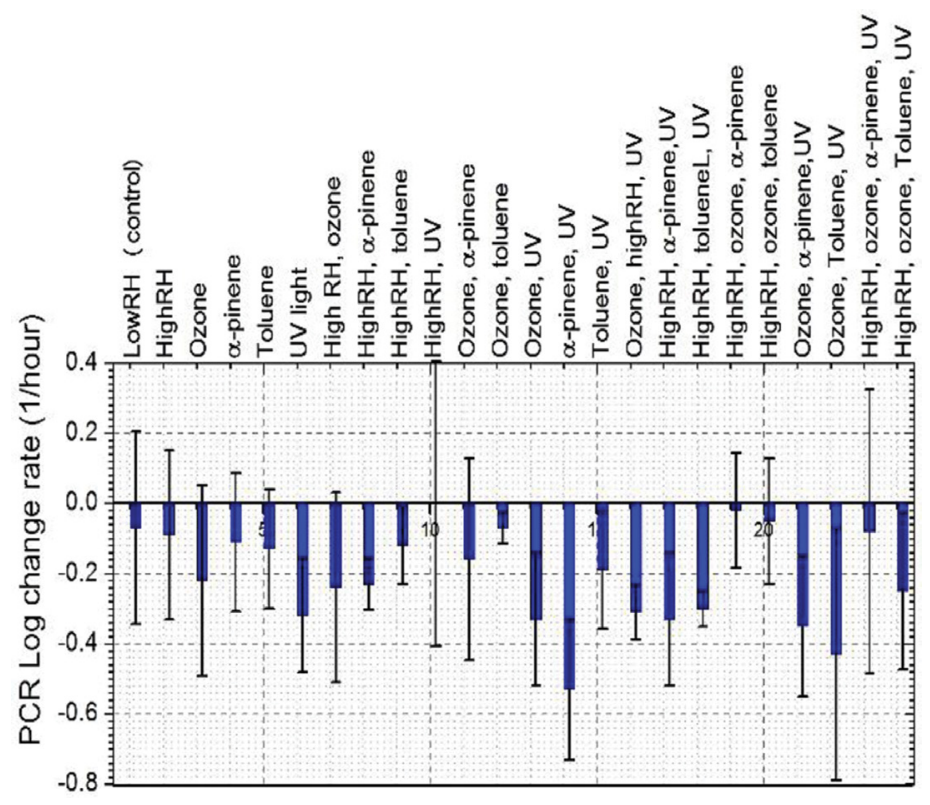

Figure 4: Log change rate (defined in eqn (1)) of PCR sensitivity for aerosolized $E$. coli cells under the different experimental conditions within the reaction chamber.

ozone did cause relatively accelerated ageing when compared to other conditions. The lack of change in PCR concentration as a function of humidity indicates that the changes in viability are likely occurring on the surface, or as a function of $\mathrm{pH}$ in the particle, instead of compromising the genomic DNA. Throughout testing, the PCR results are relatively noisy compared to the changes in viability. Since the DNA or RNA is relatively protected, it is changing slower than the viability, which relies on reactive surface proteins and cell membranes remaining intact and functional. These small changes become even noisier when normalized to particle concentrations to account for variations in physical losses, especially when compared to the relatively accelerated changes in viability.

\subsection{Changes in Fluorescence Intensity and Profile}

The averaged fluorescence spectra excited by 263 and $351 \mathrm{~nm}$ laser from 200 E. coli aerosol particles at different times were measured by SPFS during these experiments. Each spectrum is the averaged data from 200 spectra recorded during a single measurement period. Only spectra collected at $t=0, t=30 \mathrm{~min}$, and then at 1 -h intervals thereafter are presented. This shows the rapid spectral changes observed in the first hour of many experiments, while limiting the overlap as the rate of change decreases over the duration of the test. In addition to the spectra, the integrated fluorescence intensity over three emission bands is plotted verses time for all measurements (shown at the bottom row in Figs. 5-7). These averaged emission bands clearly illustrate the change trends in fluorescence amplitudes. The UV263 is the integrated fluorescence band intensity from 280 to $400 \mathrm{~nm}$ when excited by a $263 \mathrm{~nm}$ laser. The Vis 263 represents the fluorescence band intensity from 400 to $580 \mathrm{~nm}$ when excited by a $263 \mathrm{~nm}$ laser. The Vis 351 is the emission band from 380 to $650 \mathrm{~nm}$ when excited by a $351 \mathrm{~nm}$ laser. 

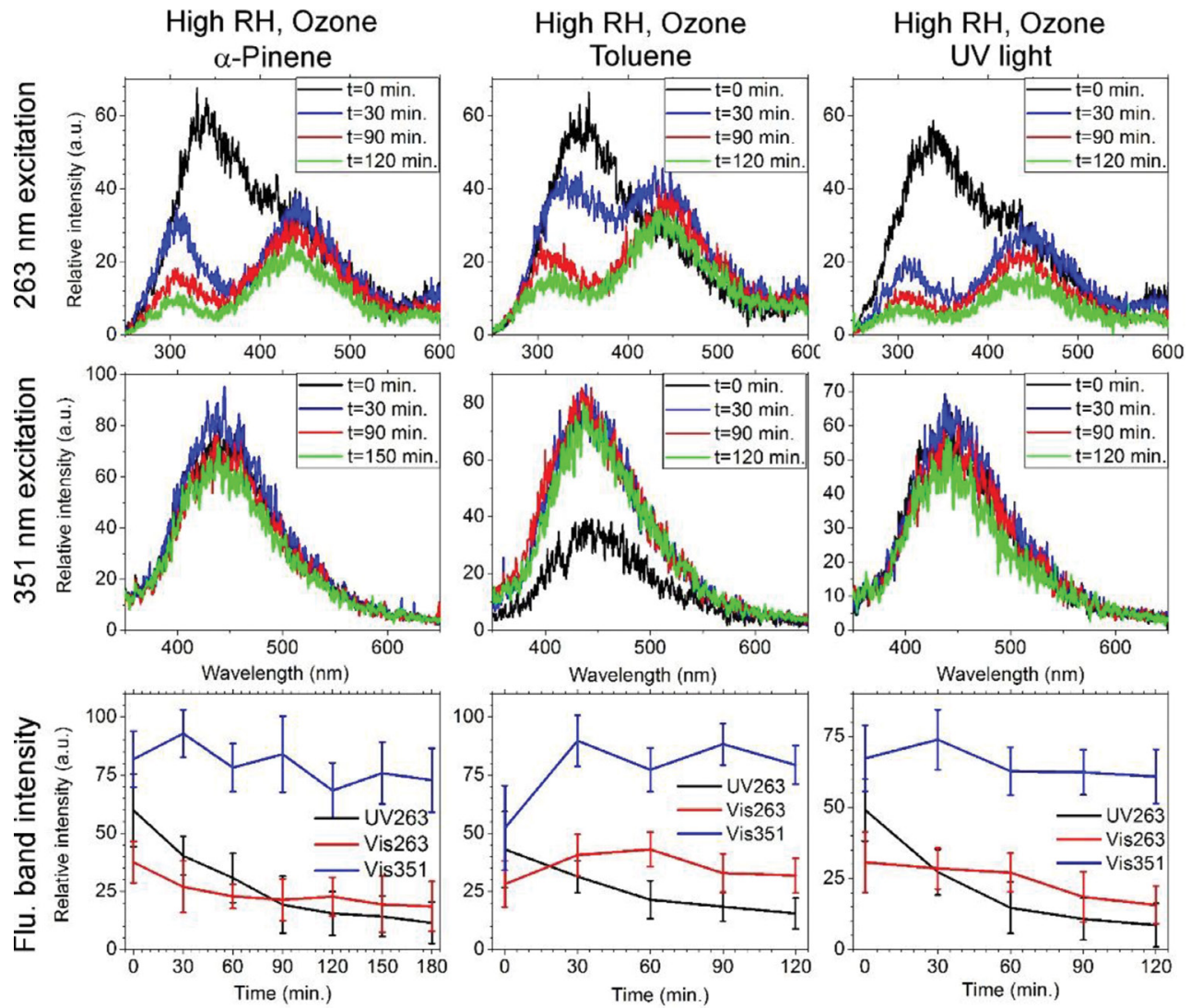

Figure 5: Time-dependent change of fluorescence spectra and the corresponding integrated band intensities from $E$. coli aerosol under various conditions in the rotational drum with three variables (High RH + Ozone + VOC or UV) applied (RH $\sim 75 \%$, ozone $\sim 100 \mathrm{ppb}, \alpha$-pinene $\sim 5 \mathrm{ppb}$, toluene $\sim 45 \mathrm{ppb}$, or UV light illumination).

Figures 5-7 show the time-dependent change of fluorescence spectra and the corresponding integrated band intensities from aerosolized $E$. coli cells under various conditions in the rotational drum with the combinations of three or four variables applied. Once ozone and high RH presented together, even with other variables added, the UV263 band always significantly decreased with some minor changes in Vis263 bands (increase or decrease). While the Vis 351 bands gave significant increase for 'ozone + high RH + toluene', and some small changes for other cases. For these conditions with UV illumination, but without 'high $\mathrm{RH}+$ ozone' presented together, all the fluorescence bands decreased, especially with a considerable decrease in the UV263 band. For the 'Ozone + VOC + UV' conditions, all the fluorescence bands decreased at a similar rate, but the spectral profiles had little change during the ageing process.

By analysing all the measured results, we noticed UV light illumination has the largest ageing effect, which drives all the fluorescence band intensities decreased. The combination of high $\mathrm{RH}$ and ozone also play an important role in the ageing process, it significantly 

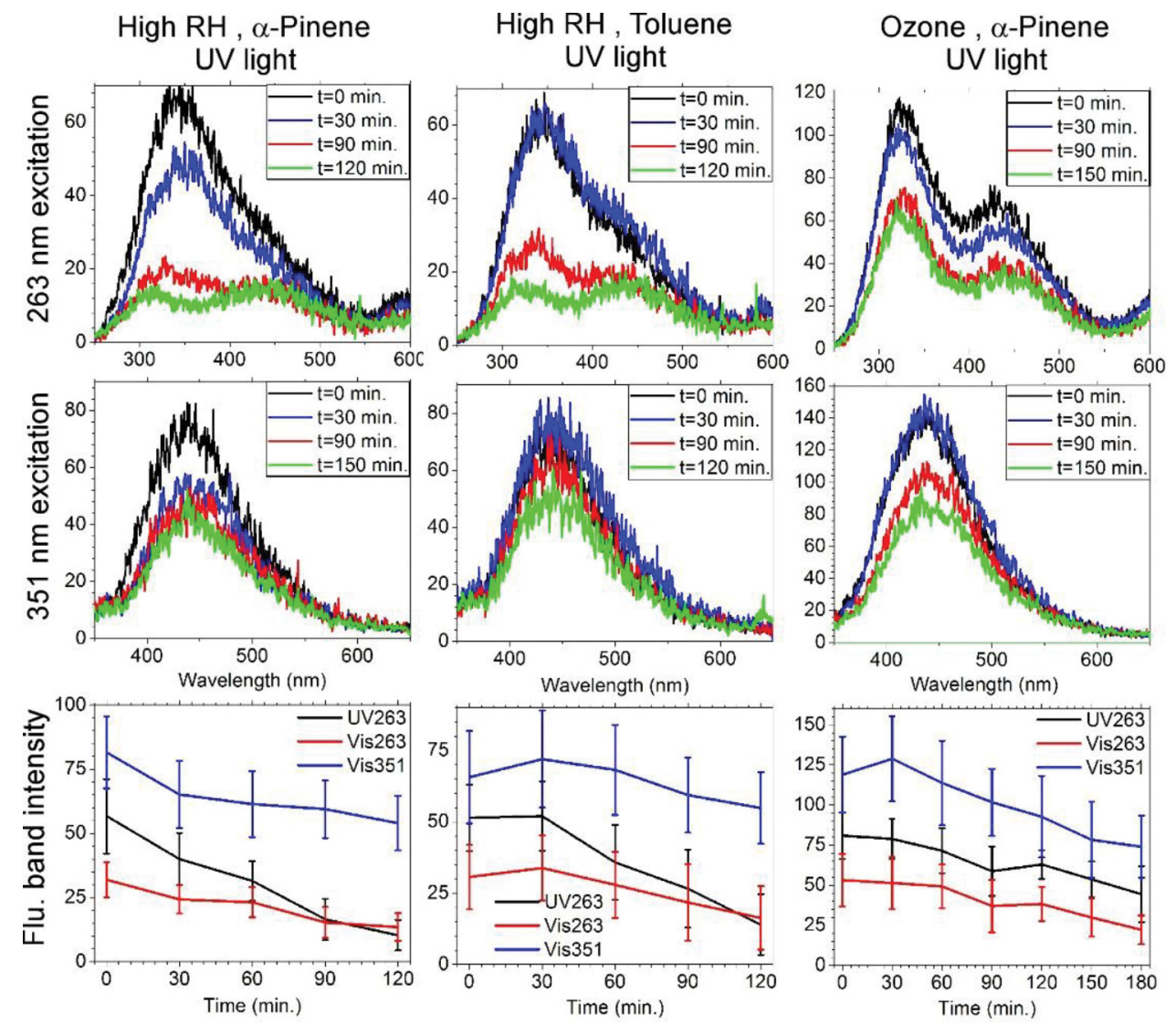

Figure 6: Time-dependent change of fluorescence spectra and the corresponding integrated band intensities from $E$. coli aerosol under various conditions with the combinations of three variables applied (RH $\sim 75 \%$, ozone $\sim 100 \mathrm{ppb}, \alpha$-pinene $\sim 5 \mathrm{ppb}$, toluene $\sim 45 \mathrm{ppb}$, or UV light illumination).

weakens the UV fluorescence, but increases the visible emission via oxidization. They showed more effects on the ageing processes as they combined with other variables. Overall, the degradation was stronger when more variables were applied.

\subsection{Comparison of the fluorescence and biological ageing results}

In order to quantitatively compare these changes under different conditions, and to make more direct comparisons with the biological ageing results, the intensity change rates of the three fluorescence bands are calculated as,

$$
\text { Avg. fluorsec.intensity change rate }=\left(I_{1}-I_{0}\right) /\left(I_{0} \times t_{1}\right) \times 100 \%
$$

where $I$ is the integrated fluorescence band intensity; $t$ is the time point for the last measurement in the whole test, which is equal to the ageing time of aerosol within the chamber; and 

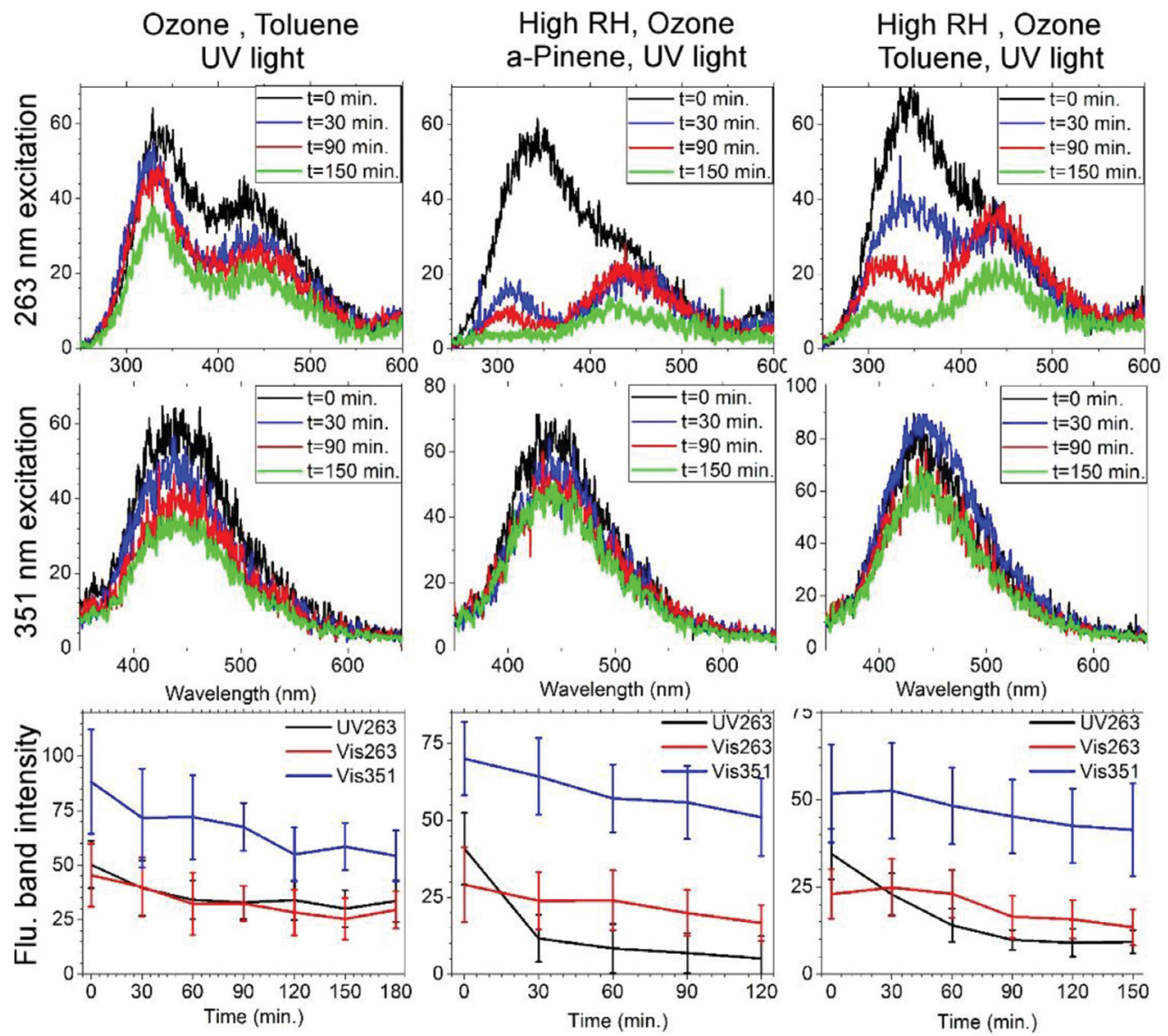

High RH, Ozone

Figure 7: Time-dependent change of fluorescence spectra and the corresponding integrated band intensities from E. coli aerosol under various conditions with the combinations of three or four variables applied (RH $\sim 75 \%$, ozone $\sim 100 \mathrm{ppb}, \alpha$-pinene $\sim 5 \mathrm{ppb}$, toluene $\sim 45 \mathrm{ppb}$, or UV light illumination).

the notation 0 and 1 indicates the initial and final aerosol sampling time point, respectively. It is important to note that this is the change averaged over the entire interaction time, which does not precisely reflect the non-linear ageing process where most ageing occurs in the first $30 \mathrm{~min}$. However, it is still valuable for comparing ageing between fluorescence, viability and PCR sensitivity. Figure 8 summarizes the average intensity change of the three fluorescence bands under the different experimental conditions. There is clearly a lot of variability, but several trends are apparent. The UV263 band typically decreases at the highest rate, and its intensity only slightly increases during limited single variable experiments (ozone or VOC). The Vis263 band typically follows the changes of UV263 (increase or decrease simultaneously) but with a smaller change rate. Vis263 and UV263 only changed in opposite directions in the ozone + high RH + toluene measurements. The Vis 351 bands have the smallest change rates, and in quite a few cases, their intensities increased especially when ozone, high RH and VOC are applied. 


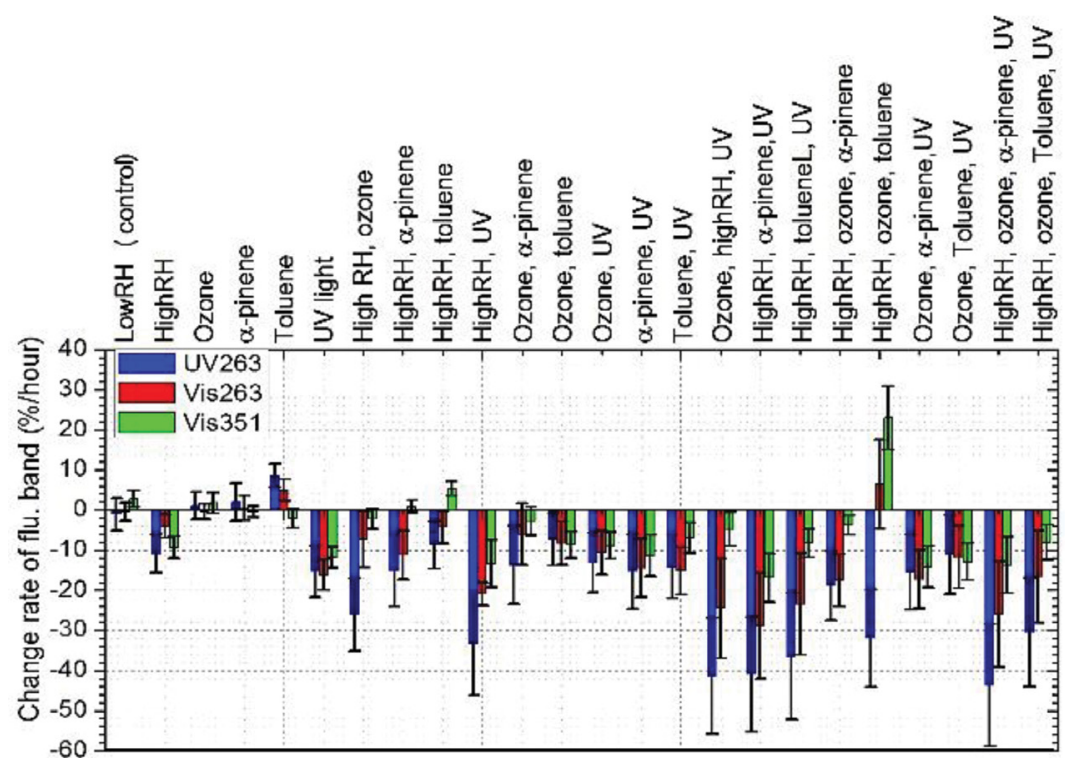

Figure 8: Time-averaged change rates of the three fluorescence bands UV263, Vis263 and Vis351 under various conditions in the rotational drum.

Comparing the rate changes in viability (Fig. 3), PCR sensitivity (Fig. 4) and intensities of fluorescence bands (Fig. 8) for the E. coli aerosols under different experimental conditions within the reaction chamber, we noticed that the changes of fluorescence do not appear directly related to a loss in viability and PCR signals. Attempt to correlate the decay rates of both fluorescence and PCR signals to the decay rate of viability produce disappointing results $\left(R^{2}=0.23,0.14,0.08\right.$ and 0.17 for UV263, Vis263, Vis351 and PCR, respectively). The UV263 fluorescence is primarily due to tryptophan and other aromatic amino acids, and as such should be a good marker for protein degradation, and therefore viability degradation. Vis263 fluorescence has some contribution of fluorescence due to amino acids, but is primarily related to flavins (flavin mononucleotide (FMN) and flavin adenine dinucleotide (FAD)), metabolic by-products. Vis351 fluorescence is dominated by NADH and its phosphate (NADPH), flavins, and metabolic products. Therefore, both Vis263 and Vis351 bands may not directly correlate to the viability of the organism when it is not active metabolically. Decay in the PCR signature should also be a good marker for DNA damage, and therefore be related to viability. However, the lack of a statistically defendable relationship between fluorescence and PCR, and viability decay rates is likely due to the variety of processes that may cause change in any of the variables, but not all. Some processes may damage DNA leading to viability loss, but not damage proteins and vice versa. It is possible that extracellular DNA was oxidized, photodegraded or destroyed during the different interacting processes in experiments and resulted in a lower genomic material available for q-PCR. This would result in the ratio between culturable cells and genomic equivalents to decrease, regardless of changes in viability. Therefore, that the use of q-PCR (measurement of total genomic equivalents in a sample) as a method of normalizing for physical loss, might not be the most appropriate method for determining biological decay of E. coli in these experiments. 


\section{SUMMARY}

The experimental results were presented for how the fluorescence spectral profile and intensity, the viability, and the PCR signature of E. coli aerosol particles change with time in the presence of one, or the combinations of two, three, or four of the following variables: relative humidity below $30 \%$ or around $\sim 75 \%$, ozone around $100 \mathrm{ppb}$, VOCs $\alpha$-pinene $(\sim 5 \mathrm{ppb})$ or toluene ( $45 \mathrm{ppb}$ ), and simulated solar UV light illumination. UV263 (280-400 nm) band always has the greatest rate of decrease except for slight increases during limited single variable experiments (with ozone or VOC). Vis263 (400-580 nm) band typically follows the changes in UV band (increase or decrease simultaneously) but with a much smaller rate of change. They did change in opposite directions only when ozone, high $\mathrm{RH}$, and toluene were applied. Vis351 (380-650 nm) band has the smallest decay rate, and in quite a few cases, their intensities increased especially when ozone, high RH and VOC are applied. Overall, all three of the viability, the PCR signature, and the fluorescence intensity of the E. coli aerosol particles decay faster when more variables were applied, and simulated solar UV light irradiation was the largest single driver of ageing, followed by the combination of high RH and ozone. Interestingly, the decay of fluorescence and PCR signals do not appear to directly correlate with a loss in viability. The results show that these changes are not special, they could happen on a typical day in any city or suburban area. Therefore, we have to reflect these ageing processes within the algorithm of bioaerosol detection systems, and hope our data observed here can provide a very rough estimation or point out a trend direction for the development of biosensors.

\section{REFERENCES}

[1] Rosenfeld, D., Sherwood, S., Wood, R. \& Donner, L., Climate effects of aerosol-cloud interactions, Science, 343, pp. 379-380, 2014.

[2] Huang, R.J., Zhang, Y., Bozzetti, C. et al., High secondary aerosol contribution to particulate pollution during haze events in China, Nature, 514, pp. 218-222, 2014.

[3] Ariya, P.A., \& Amyot, M., New directions: the role of bioaerosols in atmospheric chemistry and physics, Atmospheric Environments, 38, pp. 1231-1232, 2004.

[4] Fröhlich-Nowoisky, J., et al., Bioaerosols in the Earth system: climate, health, and ecosystem interactions, Atmospheric Research, 182 pp. 346-376, 2016.

[5] Pan, Y.L., Detection and characterization of biological and other organic-carbon aerosol particles in atmosphere using fluorescence, Journal of Quantitative Spectroscopy \& Radiative Transfer, 150, pp. 12-35, 2015.

[6] Deguillaume, L., Leriche, M., Amato, P., et al., Microbiology and atmospheric processes: chemical interaction of primary biological aerosols, Biogeosciences, 5, pp. 1073-1084, 2008.

[7] May, K.R., Druett, H.A. \& Packman, L.P., Toxicity of open air to a variety of microorganisms, Nature, 221, pp. 1146-1147, 1969.

[8] Cox, C.S., Hood, A.M. \& Baxter, J., Method for comparing concentrations of the openair factor, Applied Microbiology, 26(4), pp. 640-642, 1973.

[9] Dennis-Smither, B.J., Marshall, F.H., Miles, R.E., et al., Volatility and oxidative aging of aqueous maleic acid aerosol droplets and the dependence on relative humidity, Journal of Physical Chemistry A, 118, pp. 5680-5691, 2014.

[10] Kanaani, H., Hargreaves, M., Ristovski, Z. \& Morawska, L., Performance assessment of UVAPS: influence of fungal spore age and air exposure, Journal of Aerosol Science, 38(1), pp. 83-96, 2007. 
[11] Ignatenko, A.V., Use of the method of tryptophan fluorescence to characterize disruptions of the structure of ozonized proteins, Journal of Applied Spectroscopy, 49(1), pp. 691-695, 1988.

[12] Fujimori, E., Changes induced by ozone and ultraviolet light in type I collagen. Bovine Achilles tendon collagen versus rat tail tendon collagen. Europe Journal of Biochemistry, 152(2), 299-306 (1985).

[13] Santarpia, J.L., Pan, Y.L., Hill, S.C., et al., Changes in fluorescence spectra of bioaerosols exposed to ozone in a laboratory reaction chamber to simulate atmospheric aging, Optics Express, 20(28), pp. 29867-29881, 2012.

[14] Pan, Y.L., Santarpia, J.L., Ratnesar-Shumate, S., et al., Effects of ozone and relative humidity on fluorescence spectra of octapeptide, Journal of Quantitative Spectroscopy \& Radiative Transfer, 133, pp. 538-50, 2014.

[15] Ratnesar-Shumate, S., Pan, Y. L., Hill, S. C., et al., Fluorescence spectra and biological activity of aerosolized Bacillus spores and MS2 bacteriophage exposed to ozone at different relative humidities in a rotating drum, Journal of Quantitative Spectroscopy \& Radiative Transfer, 153, pp. 13-28, 2015

[16] Lu, J., Gerke, T. L., Buse, H. Y. \& Ashbolt, N. J., Development of an Escherichia coli K12-specific quantitative polymerase chain reaction assay and DNA isolation suited to biofilms associated with iron drinking water pipe, Journal of Water and Health, 12(4), pp. 763-771, 2014. 